Research Article

\title{
Global Existence and Extinction Singularity for a Fast Diffusive Polytropic Filtration Equation with Variable Coefficient
}

\author{
Dengming Liu $(\mathbb{D})$ and Changyu Liu \\ School of Mathematics and Computational Science, Hunan University of Science and Technology, \\ Xiangtan, Hunan 411201, China
}

Correspondence should be addressed to Dengming Liu; liudengming@hnust.edu.cn

Received 2 February 2021; Revised 4 March 2021; Accepted 1 April 2021; Published 28 April 2021

Academic Editor: Hou-Sheng Su

Copyright (c) 2021 Dengming Liu and Changyu Liu. This is an open access article distributed under the Creative Commons Attribution License, which permits unrestricted use, distribution, and reproduction in any medium, provided the original work is properly cited.

In this article, we deal with an inhomogeneous fast diffusive polytropic filtration equation. By using the energy estimate approach, Hardy-Littlewood-Sobolev inequality, and a series of ordinary differential inequalities, we prove the global existence result and obtain the conditions on the occurrence of the extinction phenomenon of the weak solution.

\section{Introduction}

Our main objectives in this article are to deal with the global existence and the extinction phenomenon of the inhomogeneous fast diffusive polytropic filtration equation:

$$
\begin{cases}|x|^{-s} u_{t}-\operatorname{div}\left(\left|\nabla u^{m}\right|^{p-2} \nabla u^{m}\right)=u^{q}, & (x, t) \in \Omega \times(0,+\infty), \\ u(x, t)=0, & (x, t) \in \partial \Omega \times(0,+\infty), \\ u(x, 0)=u_{0}(x), & x \in \Omega,\end{cases}
$$

where $\Omega \subset \mathbb{R}^{N}(N>p)$ is a bounded domain with smooth boundary $\partial \Omega, x=\left(x_{1}, \ldots, x_{N}\right) \in \Omega,|x|=\sqrt{x_{1}^{2}+\cdots+x_{N}^{2}}$, $u_{0}(x)$ is a nonnegative and bounded function with $u_{0}^{m} \in W_{0}^{1, p}(\Omega)$, and the parameters $m, s, p$, and $q$ satisfy

$$
\begin{aligned}
0 & <m \leq 1, \\
0 & <m(p-1)<1, \\
\frac{1-m}{2} & <q \leq 1, \\
0 & \leq s<\frac{N q}{q+m} .
\end{aligned}
$$

Inhomogeneous parabolic problems arise in a wide range of physical contexts (see for instance [1-3] and the references therein, where a more detailed physical background can be found). Problem (1) can be used to describe the compressible fluid flows in a homogeneous isotropic rigid porous medium with $u(x, t)$ being the density of the fluid and $\alpha(x)=|x|^{-s}$ acting as the volumetric moisture content. On the other hand parabolic models like (1), together with differential equation models, stochastic differential equations, and linear systems, are regarded as the powerful tools to solve lots of problems from control engineering, image processing, and other areas (see [4-8]). Because of the degeneracy and the singularity, problem (1) might not have classical solution in general, and hence, we introduce definition of the weak solution as follows.

Definition 1. By a local weak solution to problem (1), we understand a function $u \in \mathbb{S} \stackrel{\text { def }}{=}\left\{u \in C\left(0, T ; L^{1}(\Omega)\right), u \in\right.$ $\left.L^{2 q}(\Omega \times(0, T)) \cap L^{2}(\Omega \times(0, T)), \nabla u^{m} \in L^{p}(\Omega \times(0, T))\right\}$ for some $T>0$, which moreover satisfies the following assumptions:

(i) For any $0 \leq \phi \in \widetilde{\mathfrak{S}} \stackrel{\text { def }}{=}\left\{\phi \in \mathbb{S},\left.\phi\right|_{\partial \Omega}=0\right\} \quad$ and $0<t_{1}<t_{2}<T$, one has 


$$
\int_{\Omega}|x|^{-s} u_{t} \phi \mathrm{d} x+\int_{\Omega}\left|\nabla u^{m}\right|^{p-2} \nabla u^{m} \cdot \nabla \phi \mathrm{d} x=\int_{\Omega} u^{q} \phi \mathrm{d} x .
$$

(ii) $u(x, t) \longrightarrow u_{0}(x)$ as $t \longrightarrow 0$ with convergence in $L^{1}(\Omega)$.

In the past few decades, many mathematicians have studied the global existence, blow-up, and extinction phenomena of the following parabolic equation:

$$
|x|^{-s} u_{t}-\operatorname{div}\left(\left|\nabla u^{m}\right|^{p-2} \nabla u^{m}\right)=f(u), \quad(x, t) \in \Omega \times(0,+\infty),
$$

subject to various assumptions (see [9-23] and the references therein). For the case $s=0$, the authors in [24-26] concerned with the global existence and blow-up properties of the solutions to problem (4) with $m=1$ and $p \geq 2$. Yuan et al. [27] considered problem (4) with $m=1$ and $f(u)=0$ and showed that the solution of problem (4) vanishes in finite time if and only if $1<p<2$. Gu [28] studied problem (4) with $m=1$ and $f(u)=-u^{q}$ and claimed that the necessary and sufficient condition on the occurrence of extinction phenomenon is $p \in(1,2)$ or $q \in(0,1)$. Tian and $\mathrm{Mu}$ [29] and Jin and Yin [30] studied problem (4) with $m=1$ and $f(u)=\lambda u^{q}$ with $\lambda>0$ and showed that $q=p-1$ is the critical extinction exponent of the solutions. When $s=0$ and $f(u)=\lambda u^{q}$ with $\lambda>0$, Jin et al. [31] and Zhou and $\mathrm{Mu}$ [32] concluded that the critical extinction exponent of the solution to problem (1) is $q=m(p-1)$. Compared with $s=0$, there are few literatures for the case $s>0$. By Hardy inequality and potential well method, Tan [33] obtained the global existence and blow-up results of problem (4) with $s=$ 2 and $m=1$. Wang [34] generated the results in [33] to the case $0<s \leq 2$. Zhou generated the results in [33] to the case $s \geq 2$ and gave the global existence and blow-up results for (4) with $p=2$ and $m=1$ in $[35,36]$, respectively. To the best knowledge of us, there is little work on the global existence and extinction behavior of problem (1). In a recent paper, Deng and Zhou [37] considered the special case $m=1$ and analysed the effect of the singular potential on the global existence and extinction behavior of the solutions.
In order to state well our results, we first introduce some definitions, fundamental facts, and useful symbols. Since $\Omega$ is a bounded domain in $\mathbb{R}^{N}$, then there is a ball $B(0, R) \subset \mathbb{R}^{N}$ centered at 0 with radius

$$
R=\sup _{x \in \Omega} \sqrt{x_{1}^{2}+\cdots+x_{N}^{2}},
$$

such that $\Omega \subseteq B(0, R)$.

We denote the norm of $L^{r}(\Omega)$ by $\|\cdot\|_{r}$ and the norm of $W^{1, r}(\Omega)$ by $\|\cdot\|_{W^{1, r}(\Omega)}$; that is, for any $\phi \in L^{r}(\Omega)$,

$$
\|\phi\|_{r}= \begin{cases}\left(\int_{\Omega}|\phi(x)|^{r} \mathrm{~d} x\right)^{1 / r} & \text { if } 1 \leq r<+\infty \\ \underset{x \in \Omega}{\operatorname{ess} \sup _{x}|\phi(x)|,} & \text { if } r=+\infty\end{cases}
$$

and for any $\phi \in W^{1, r}(\Omega),\|\phi\|_{W^{1, r}(\Omega)}=\sqrt{[r]\|\phi\|_{r}^{r}+\|\nabla \phi\|_{r}^{r}}$. According to Poincaré's inequality, one can see that $\|\nabla(\cdot)\|_{r}$ is equivalent to $\|\cdot\|_{W^{1, r}(\Omega)}$ in $W_{0}^{1, r}(\Omega)$, and hence, we equip $W_{0}^{1, r}(\Omega)$ with the norm $\|\phi\|^{\text {def }}=\|\nabla \phi\|_{r}$.

Let $u(x, t)$ be a weak solution of problem (1). Define an energy functional as the following form:

$$
E(u)=\frac{1}{p} \int_{\Omega}\left|\nabla u^{m}\right|^{p} \mathrm{~d} x-\frac{m}{m+q} \int_{\Omega} u^{m+q} \mathrm{~d} x .
$$

Then, by (3), one can easily show that

$$
\frac{\partial E(u)}{\partial t}=-m \int_{\Omega} u^{m-1}\left(u_{t}\right)^{2} \mathrm{~d} x \leq 0,
$$

which tells us that $E(u)$ is nonincreasing with respect to $t$.

We state our main results as follows.

Theorem 1. Suppose that the parameters $m, p, q$, and $s$ satisfy (2), and the initial data $u_{0}(x)$ are a nonnegative and bounded function with $u_{0}^{m} \in W_{0}^{1, p}(\Omega)$. Let $u(x, t)$ be a solution of problem (1). Then, the maximal existence time of $u(x, t)$ is $T=+\infty$; that is, $u(x, t)$ is a global solution. Moreover,

(i) If $0<\max \{m(p-1),(1-m) / 2\}<q \leq 1 \quad$ and $0 \leq s \leq \min \{p, N q /(m+q)\}$ and there is a constant $a>\max \{(m+1-\alpha m) /(m \alpha-m p),-(1 / p)\}$ with $\alpha=$ $p(N-s) /(N-p)$ such that

$$
\begin{aligned}
& \left(\int_{\Omega}|x|^{-s} u_{0}^{m(p a+1)+1} \mathrm{~d} x\right)^{(q-m(p-1)) /(m(p a+1)+1)} \\
& \quad \leq \frac{p a+1}{2(a+1)^{p}} R^{-(s[m(p a+1)+q]) /(m(p a+1)+1)}|\Omega|^{(q-1) /(m(p a+1)+1)} \kappa_{1}^{((N-p) /(N-s))-(m p(a+1) /(m(p a+1)+1))} \kappa_{2}^{-((N-p) /(N-s))}
\end{aligned}
$$

where $R, \kappa_{1}$, and $\kappa_{2}$ are given by (5), (12), and Lemma 2 , respectively, then the solution $u(x, t)$ of problem (1) vanishes in finite time.

(ii) If $0<((1-m) / 2)<q \leq m(p-1)<1$ and

$$
\left\{\begin{array}{l}
\int_{\Omega}|x|^{-s} u_{0}^{m+1} \mathrm{~d} x>0 \text { and } E\left(u_{0}\right) \leq 0, \quad \text { when } q=m(p-1) \\
\int_{\Omega}|x|^{-s} u_{0}^{m+1} \mathrm{~d} x>0 \text { and } E\left(u_{0}\right)<0,
\end{array}\right.
$$


where

$$
E\left(u_{0}\right)=\frac{1}{p} \int_{\Omega}\left|\nabla u_{0}^{m}\right|^{p} \mathrm{~d} x-\frac{m}{m+q} \int_{\Omega} u_{0}^{m+q} \mathrm{~d} x,
$$

then the solution $u(x, t)$ of problem (1) does not possess extinction phenomenon.

The rest of this article is organized as follows. In Section 2 , we collect some useful auxiliary lemmas. The last section is mainly focused on the global existence and the conditions on the occurrence of the extinction phenomenon of the solution. By Hardy-Littlewood-Sobolev inequality and some ordinary differential inequalities, the proof of Theorem 1 will be given in Section 3 .

\section{Preliminaries}

In this section, as preliminaries, we collect some well-known results, which play an important role in our proof of Theorem 1 .

Lemma 1 (see [37]). Suppose $N>s$ and $\Omega \subset \mathbb{R}^{N}$ is a bounded domain. Then, we have

$$
\begin{aligned}
\kappa_{1} \stackrel{\text { def }}{=} \int_{\Omega}|x|^{-s} \mathrm{~d} x & \leq \int_{B(0, R)}|x|^{-s} \mathrm{~d} x=\int_{0}^{R}\left[\int_{\partial B(0, r)}|x|^{-s} \mathrm{~d} S(x)\right] \mathrm{d} r \\
& =\omega_{N} \int_{0}^{R} r^{-s} r^{N-1} \mathrm{~d} r=\frac{\omega_{N}}{N-s} R^{N-s}<+\infty,
\end{aligned}
$$

where $B(0, R)$ is the ball in $\mathbb{R}^{N}$ centered at 0 with radius $R=\sup _{x \in \Omega} \sqrt{x_{1}^{2}+\cdots+x_{N}^{2}}$ satisfying $\Omega \subset B(0, R)$ and

$$
\omega_{N}=\frac{N \pi^{N / 2}}{\Gamma((N / 2)+1)}
$$

denotes the surface area of the unit sphere $\partial B(0,1)$, and $\Gamma$ is the usual Gamma function.

Lemma 2 (see [38]). Suppose $N \geq 2,1<\mu<N, 0 \leq \vartheta \leq \mu$, and $\sigma=\mu(N-\vartheta) /(N-\mu)$. Then, there is a positive constant $\kappa_{2}=$ $\kappa_{2}(\mu, \vartheta, N)$ such that

$$
\int_{\Omega} \frac{|u(x)|^{\sigma}}{|x|^{9}} \mathrm{~d} x \leq \kappa_{2}\left(\int_{\Omega}|\nabla u|^{\mu} \mathrm{d} x\right)^{(N-9) /(N-\mu)}
$$

holds for any $u \in W_{0}^{1, \mu}(\Omega)$, where $\Omega \subset \mathbb{R}^{N}$ is a bounded domain.

Lemma 3 (see [39]). Assume $\theta, \delta$, and $\beta$ are positive constants. Let $y(t)$ be a nonnegative absolutely continuous function satisfying

$$
\frac{\mathrm{d} y}{\mathrm{~d} t}+\delta y^{\theta}(t) \geq \beta, \quad t>0 .
$$

Then, we have

$$
y(t) \geq \min \left\{y(0),\left(\frac{\beta}{\delta}\right)^{1 / \theta}\right\}
$$

Lemma 4 (see [40]). Suppose $0<k<r \leq 1$. Let $y(t)$ be the solution of the ordinary differential inequality:

$$
\left\{\begin{array}{l}
\frac{\mathrm{d} y}{\mathrm{~d} t}+C y^{k} \leq \gamma y^{r}, \quad t>0, \\
y(0)=y_{0}>0,
\end{array}\right.
$$

where $C>0$ and $0<\gamma<\left(C y_{0}^{k-r} / 2\right)$. Then, there are two positive constants $\eta$ and $\xi$ such that, for $t \geq 0$,

$$
0 \leq y(t) \leq \xi e^{-\eta t}
$$

\section{Proof of Theorem 1}

In this section, we will give the proof of the global existence result and the conditions on the occurrence of the extinction phenomenon of the solution $u(x, t)$.

Case 1. If $((1-m) / 2)<q<1$. Taking the test function $\phi=$ $u^{m}(x, t)$ in (3), and using Hölder's inequality, one has

$$
\begin{aligned}
\frac{1}{m+1} \frac{\mathrm{d}}{\mathrm{d} t} \int_{\Omega}|x|^{-s} u^{m+1} \mathrm{~d} x+\left\|u^{m}\right\|^{p} & =\int_{\Omega} u^{q+m} \mathrm{~d} x=\int_{\Omega}|x|^{-(s(q+m) /(1+m))} u^{q+m}|x|^{(s(q+m) /(1+m))} \mathrm{d} x \\
& \leq\left(\int_{\Omega}|x|^{-s} u^{m+1} \mathrm{~d} x\right)^{(m+q) /(m+1)}\left(\int_{\Omega}|x|^{s(m+q) /(1-q)} \mathrm{d} x\right)^{(1-q) /(m+1)} \\
& \leq \kappa_{3}\left(\int_{\Omega}|x|^{-s} u^{m+1} \mathrm{~d} x\right)^{(m+q) /(m+1)},
\end{aligned}
$$

which implies that 
$\frac{\mathrm{d}}{\mathrm{d} t} \int_{\Omega}|x|^{-s} u^{m+1} \mathrm{~d} x \leq \kappa_{3}(m+1)\left(\int_{\Omega}|x|^{-s} u^{m+1} \mathrm{~d} x\right)^{(m+q) /(m+1)}, \quad$ where

$$
\kappa_{3}=\left(\int_{B(0, R)}|x|^{s(q+m) /(1-q)} \mathrm{d} x\right)^{(1-q) /(1+m)}=\left(\frac{\omega_{N}(1-q)}{s(q+m)+N(1-q)} R^{(s(q+m)+N(1-q)) /(1-q)}\right)^{(1-q) /(1+m)}
$$

Integrating (20) from 0 to $t$, one gets

$$
\int_{\Omega}|x|^{-s} u^{m+1} \mathrm{~d} x \leq\left[\kappa_{3}(1-q) t+\left(\int_{\Omega}|x|^{-s} u_{0}^{m+1} \mathrm{~d} x\right)^{(1-q) /(m+1)}\right]^{(m+1) /(1-q)} .
$$

From (19) and (22), it follows that

$\|u\|_{q+m}^{q+m} \leq \kappa_{3}\left[\kappa_{3}(1-q) t+\left(\int_{\Omega}|x|^{-s} u_{0}^{m+1} \mathrm{~d} x\right)^{(1-q) /(m+1)}\right]^{(m+q) /(1-q)}$.
On the other hand, taking the test function $\phi=\left(u^{m}\right)_{t}$ in (3), then by using Cauchy's inequality with $\varepsilon$ and Hölder's inequality, one can obtain

$$
\begin{aligned}
& \frac{4 m}{(m+1)^{2}} \int_{\Omega}|x|^{-s}\left[\left(u^{(m+1) / 2}\right)_{t}\right]^{2} \mathrm{~d} x+\frac{1}{p} \frac{\mathrm{d}}{\mathrm{d} t}\left\|u^{m}\right\|^{p} \\
& =\frac{2 m}{m+1} \int_{\Omega}|x|^{s / 2} u^{(m+2 q-1) / 2}|x|^{-(s / 2)}\left(u^{(m+1) / 2}\right)_{t} \mathrm{~d} x \\
& \leq \frac{2 m \varepsilon}{m+1} \int_{\Omega}|x|^{-s}\left[\left(u^{(m+1) / 2}\right)_{t}\right]^{2} \mathrm{~d} x+\frac{m}{2 \varepsilon(m+1)} \int_{\Omega}|x|^{s} u^{m+2 q-1} \mathrm{~d} x \\
& \leq \frac{2 m \varepsilon}{m+1} \int_{\Omega}|x|^{-s}\left[\left(u^{(m+1) / 2}\right)_{t}\right]^{2} \mathrm{~d} x+\frac{m}{2 \varepsilon(m+1)}\left(\int_{\Omega}|x|^{s(q+m) /(1-q)} \mathrm{d} x\right)^{(1-q) /(q+m)}\left(\int_{\Omega} u^{q+m} \mathrm{~d} x\right)^{(m+2 q-1) /(q+m)}
\end{aligned}
$$

Let $\varepsilon$ be sufficiently small to ensure that $\left(4 m /(m+1)^{2}\right)-(2 m \varepsilon /(m+1)) \geq 0$, then by (21), (23), and (24), one has

$$
\frac{\mathrm{d}}{\mathrm{d} t}\left\|u^{m}\right\|^{p} \leq \frac{m p \kappa_{3}^{2}}{2 \varepsilon(m+1)}\left[\kappa_{3}(1-q) t+\left(\int_{\Omega}|x|^{-s} u_{0}^{m+1} \mathrm{~d} x\right)^{(1-q) /(m+1)}\right]^{(m+2 q-1) /(1-q)} .
$$

Integrating (25) from 0 to $t$ yields that 


$$
\begin{aligned}
\left\|u^{m}\right\|^{p} \leq & \left\|u_{0}^{m}\right\|^{p}+\frac{m p \kappa_{3}^{2}}{2 \varepsilon(m+1)} \int_{0}^{t}\left[\kappa_{3}(1-q) \tau+\left(\int_{\Omega}|x|^{-s} u_{0}^{m+1} \mathrm{~d} x\right)^{(1-q) /(m+1)}\right]^{(m+2 q-1) /(1-q)} \mathrm{d} \tau \\
\leq & \left\|u_{0}^{m}\right\|^{p}-\frac{m p \kappa_{3}}{2 \varepsilon(m+1)(m+q)}\left(\int_{\Omega}|x|^{-s} u_{0}^{m+1} \mathrm{~d} x\right)^{(m+q) /(m+1)} \\
& +\frac{m p \kappa_{3}}{2 \varepsilon(m+1)(m+q)}\left(\kappa_{3}(1-q) t+\left(\int_{\Omega}|x|^{-s} u_{0}^{m+1} \mathrm{~d} x\right)^{(1-q) /(m+1)}\right)^{(m+q) /(1-q)}
\end{aligned}
$$

which means that the solution $u(x, t)$ of the problem (1) is global.

Case 2. If $q=1$, taking the test function $\phi=u^{m}(x, t)$ in (3), then we can see that

$$
\begin{aligned}
& \frac{1}{m+1} \frac{\mathrm{d}}{\mathrm{d} t} \int_{\Omega}|x|^{-s} u^{m+1} \mathrm{~d} x+\left\|u^{m}\right\|^{p} \\
= & \int_{\Omega}|x|^{-s} u^{m+1}|x|^{s} \mathrm{~d} x \leq R^{s} \int_{\Omega}|x|^{-s} u^{m+1} \mathrm{~d} x,
\end{aligned}
$$

which tells us that

$$
\begin{aligned}
\int_{\Omega}|x|^{-s} u^{m+1} \mathrm{~d} x & \leq e^{(m+1) R^{s} t} \int_{\Omega}|x|^{-s} u_{0}^{m+1} \mathrm{~d} x, \\
\|u\|_{m+1}^{m+1} & \leq R^{s} e^{(m+1) R^{s} t} \int_{\Omega}|x|^{-s} u_{0}^{m+1} \mathrm{~d} x .
\end{aligned}
$$

On the other hand, taking the test function $\phi=\left(u^{m}\right)_{t}$ in (3), then Cauchy's inequality with $\varepsilon$ leads to

$$
\begin{aligned}
& \frac{4 m}{(m+1)^{2}} \int_{\Omega}|x|^{-s}\left[\left(u^{(m+1) / 2}\right)_{t}\right]^{2} \mathrm{~d} x+\frac{1}{p} \frac{\mathrm{d}}{\mathrm{d} t}\left\|u^{m}\right\|^{p} \\
= & \frac{2 m}{m+1} \int_{\Omega}|x|^{s / 2} u^{(m+1) / 2}|x|^{-(s / 2)}\left(u^{(m+1) / 2}\right)_{t} \mathrm{~d} x \\
\leq & \frac{2 m \varepsilon}{m+1} \int_{\Omega}|x|^{-s}\left[\left(u^{(m+1) / 2}\right)_{t}\right]^{2} \mathrm{~d} x+\frac{m}{2 \varepsilon(m+1)} \int_{\Omega}|x|^{s} u^{m+1} \mathrm{~d} x \\
\leq & \frac{2 m \varepsilon}{m+1} \int_{\Omega}|x|^{-s}\left[\left(u^{(m+1) / 2}\right)_{t}\right]^{2} \mathrm{~d} x+\frac{m R^{s}}{2 \varepsilon(m+1)} \int_{\Omega} u^{m+1} \mathrm{~d} x .
\end{aligned}
$$

Choosing $\varepsilon \in(0,(2 /(m+1)))$ to guarantee that $\left(4 m /(m+1)^{2}\right)-(2 \lambda m \varepsilon /(m+1)) \geq 0$, then by (29), one has

$$
\frac{\mathrm{d}}{\mathrm{d} t}\left\|u^{m}\right\|^{p} \leq \frac{m p R^{2 s}}{2 \varepsilon(m+1)} e^{(m+1) R^{s} t} \int_{\Omega}|x|^{-s} u_{0}^{m+1} \mathrm{~d} x
$$

which implies that

$$
\left\|u^{m}\right\|^{p} \leq\left\|u_{0}^{m}\right\|^{p}+\frac{m p R^{s}}{2 \varepsilon(m+1)^{2}}\left(e^{(m+1) R^{s} t}-1\right) \int_{\Omega}|x|^{-s} u_{0}^{m+1} \mathrm{~d} x
$$

Then, the proof of the global existence result is complete.

Now, we take our attention to the extinction singularity of the solution $u(x, t)$ to problem (1). We denote $\alpha=p(N-s) /(N-p)$. Noticing that $0 \leq s<p$, we can verify that $\alpha>p$. Let $a$ be a constant satisfying

$$
a>\max \left\{\frac{m+1-\alpha m}{m \alpha-m p},-\frac{1}{p}\right\} \text {. }
$$

From (32), it follows that

$$
0<\frac{m(p a+1)+1}{\alpha m(a+1)}<1 .
$$

Selecting the test function $\phi=u^{m(p a+1)}(x, t)$ in (3), one has

$$
\frac{1}{m(p a+1)+1} \frac{\mathrm{d}}{\mathrm{d} t} \int_{\Omega}|x|^{-s} u^{m(p a+1)+1} \mathrm{~d} x+\frac{p a+1}{(a+1)^{p}} \int_{\Omega}\left|\nabla u^{m(a+1)}\right|^{p} \mathrm{~d} x=\int_{\Omega} u^{m(p a+1)+q} \mathrm{~d} x
$$

Making use of Hölder's inequality, one can find that 


$$
\begin{aligned}
\int_{\Omega}|x|^{-s} u^{m(p a+1)+1} \mathrm{~d} x & =\int_{\Omega}|x|^{-(s[m(p a+1)+1] / \alpha m(a+1))} u^{m(a+1) \cdot((m(p a+1)+1) / m(a+1))}|x|^{-s(1-((m)(p a+1)+1) / \alpha m(a+1)))} \mathrm{d} x \\
& \leq\left(\int_{\Omega}|x|^{-s} u^{\alpha m(a+1)} \mathrm{d} x\right)^{(m(p a+1)+1) / \alpha m(a+1)}\left(\int_{\Omega}|x|^{-s} \mathrm{~d} x\right)^{1-((m(p a+1)+1) / \alpha m(a+1))} \\
& =\kappa_{1}^{1-((m(p a+1)+1) / \alpha m(a+1))}\left(\int_{\Omega}|x|^{-s} u^{\alpha m(a+1)} \mathrm{d} x\right)^{(m(p a+1)+1) / \alpha m(a+1)},
\end{aligned}
$$

where $\kappa_{1}$ is the same as that in $(12)$. Since $0<m(p-1)<1$ and $0<m \leq 1$, one can deduce that $1<p<1+(1 / m)$. This together with the assumption $N>p$ one has $1<p<\min \{N, 1+(1 / m)\}$. Meanwhile, recalling that $0 \leq s<\min \{p,(N q /(q+m))\}$, then it follows from Lemma 2 that

$$
\int_{\Omega}|x|^{-s} u^{\alpha m(a+1)} \mathrm{d} x=\int_{\Omega}|x|^{-s} u^{m(a+1) \cdot(p(N-s) /(N-p))} \mathrm{d} x \leq \kappa_{2}\left(\int_{\Omega}\left|\nabla u^{m(a+1)}\right|^{p} \mathrm{~d} x\right)^{(N-s) /(N-p)},
$$

where $\kappa_{2}$ is given in Lemma 2. Combining (35) with (32), one sees

$$
\begin{aligned}
\int_{\Omega}|x|^{-s} u^{m(p a+1)+1} \mathrm{~d} x & \leq \kappa_{1}^{1-((m(p a+1)+1) / \alpha m(a+1))} \kappa_{2}^{((m(p a+1)+1) / \alpha m(a+1))}\left(\int_{\Omega}\left|\nabla u^{m(a+1)}\right|^{p} \mathrm{~d} x\right)^{((N-s)[m(p a+1)+1]) /(\alpha m(a+1)(N-p))} \\
& =\kappa_{1}^{1-((m(p a+1)+1) / \alpha m(a+1))} \kappa_{2}^{((m(p a+1)+1) / \alpha m(a+1))}\left(\int_{\Omega}\left|\nabla u^{m(a+1)}\right|^{p} \mathrm{~d} x\right)^{((m(p a+1)+1) / m p(a+1))}
\end{aligned}
$$

Exploiting (34) and (37), one can arrive at

$$
\begin{aligned}
& \frac{1}{m(p a+1)+1} \frac{\mathrm{d}}{\mathrm{d} t} \int_{\Omega}|x|^{-s} u^{m(p a+1)+1} \mathrm{~d} x \\
& \quad+\frac{p a+1}{(a+1)^{p}} \kappa_{1}^{((N-p) /(N-s))-(m p(a+1) /(m(p a+1)+1))} \kappa_{2}^{-((N-p) /(N-s))}\left(\int_{\Omega}|x|^{-s} u^{m(p a+1)+1} \mathrm{~d} x\right)^{m p(a+1) /(m(p a+1)+1)} \\
& \quad \leq \int_{\Omega} u^{q+m(p a+1)} \mathrm{d} x
\end{aligned}
$$

In what follows, for the sake of simplicity, we denote $y(t)=\int_{\Omega}|x|^{-s} u^{m(p a+1)+1} \mathrm{~d} x$, and $C_{1}=[m(p a+1)$ $+1]\left((p a+1) /(a+1)^{p}\right) \kappa_{1}^{((N-p) /(N-s))-(m p(a+1) /(m(p a+1)+1)}$

If $\max \{((1-m) / 2), m(p-1)\}<q=1$, then from (38), one can immediately know that

$$
\frac{\mathrm{d} y}{\mathrm{~d} t}+C_{1} y^{m p(a+1) /(m(p a+1)+1)}(t) \leq C_{2} y(t),
$$

where $C_{2}=[m(p a+1)+1] R^{s}$. Remembering that $0<m(p-1)<1$ and (32), one can check that $0<(m p(a+1) /(m(p a+1)+1))<1$. If $2 C_{2}<C_{1}$ $y^{(m(p-1)-1) /(m(p a+1)+1)}(0)$, then Lemma 4 tells us that there are two positive constants $\eta_{1}$ and $\xi_{1}$ satisfying

$$
0 \leq y(t) \leq \xi_{1} e^{-\eta_{1} t}
$$

Putting $T_{0}=\max \left\{0,\left((m(p a+1)+1) / \eta_{1}[1-m \quad(p-\right.\right.$ 1)] $\left.) \ln \left(\left(2 C_{2} / C_{1}\right) \xi_{1}^{(1-m(p-1)) /(m(p a+1)+1)}\right)\right\}$, then for any $t>T_{0}$, (40) leads to

$$
2 C_{2} y^{(1-m(p-1)) /(m(p a+1)+1)}(t)<C_{1},
$$

which together with (39) yields

$$
\frac{\mathrm{d} y}{\mathrm{~d} t}+\frac{C_{1}}{2} y^{m p(a+1) /(m(p a+1)+1)} \leq 0, \quad t \geq T_{0}
$$

Integrating above inequality from $T_{0}$ to $t$ leads to 


$$
y^{(1-m(p-1)) /(m(p a+1)+1)}(t) \leq y^{(1-m(p-1)) /(m(p a+1)+1)}\left(T_{0}\right)-\frac{C_{1}[1-m(p-1)]}{2[m(p a+1)+1]}\left(t-T_{0}\right), \quad t \geq T_{0} .
$$

The above inequality means that

$$
\lim _{t \longrightarrow T_{1}} y(t)=\lim _{t \longrightarrow T_{1}} \int_{\Omega}|x|^{-s} u^{m(p a+1)+1} \mathrm{~d} x=0,
$$

where

$$
T_{1}=T_{0}+\frac{2[m(p a+1)+1]}{C_{1}[1-m(p-1)]} y^{\frac{1-m(p-1)}{m(p a+1)+1}}\left(T_{0}\right)
$$

If $\max \{((1-m) / 2), m(p-1)\}<q<1$. In view of Hölder's inequality, one has

$$
\begin{aligned}
\int_{\Omega} u^{m(p a+1)+q} \mathrm{~d} x & \leq|\Omega|^{(1-q) /(m(p a+1)+1)}\left(\int_{\Omega} u^{m(p a+1)+1} \mathrm{~d} x\right)^{(m(p a+1)+q) /(m(p a+1)+1)} \\
& \leq|\Omega|^{(1-q) /(m(p a+1)+1)} R^{s[m(p a+1)+q] /(m(p a+1)+1)}\left(\int_{\Omega}|x|^{-s} u^{m(p a+1)+1} \mathrm{~d} x\right)^{(m(p a+1)+q) /(m(p a+1)+1)}
\end{aligned}
$$

Combining (38) with (46), one can conclude that

$$
\frac{\mathrm{d} y}{\mathrm{~d} t}+C_{1} y^{m p(a+1) /(m(p a+1)+1)}(t) \leq C_{3} y^{(m(p a+1)+q) /(m(p a+1)+1)}(t),
$$

where

$$
C_{3}=\lambda[m(p a+1)+1]|\Omega|^{(1-q) /(m(p a+1)+1)} R^{s[m(p a+1)+q] /(m(p a+1)+1)} .
$$

Recalling that $0<m(p-1)<q<1$ and (32), one can check that

$$
0<\frac{m p(a+1)}{m(p a+1)+1}<\frac{m(p a+1)+q}{m(p a+1)+1}<1 .
$$

Then, by (47) and Lemma 4, one knows that there are two positive constants $\eta_{2}$ and $\xi_{2}$ satisfying

$$
0 \leq y(t) \leq \xi_{2} e^{-\eta_{2} t}
$$

provided that $2 C_{3} y^{(q-m(p-1)) /(m(p a+1)+1)}(0)<C_{1}$. Setting $T_{2}=\max \left\{0,\left((m(p a+1)+1) / \eta_{2}[q-m(p-1)]\right) \ln \left(\left(2 C_{3} /\right.\right.\right.$ $\left.\left.\left.C_{1}\right) \xi_{2}^{(q-m(p-1)) /(m(p a+1)+1)}\right)\right\}$, then for any $t>T_{1}$, (50) leads to

$$
2 C_{3} y^{(q-m(p-1)) /(m(p a+1)+1)}(t)<C_{1},
$$

which together with (47) yields

$$
\frac{\mathrm{d} y}{\mathrm{~d} t}+\frac{C_{1}}{2} y^{m p(a+1) /(m(p a+1)+1)} \leq 0, \quad t \geq T_{2} .
$$

The remainder proof is the same as the previous one in the case $q=1$, and we omit it here. Up to now, the proof of the extinction phenomenon of the solution $u(x, t)$ to problem (1) is complete.

Now, we begin to prove the nonextinction result. Denoting

$$
M(t)=\frac{1}{m+1} \int_{\Omega}|x|^{-s} u^{m+1} \mathrm{~d} x
$$

and taking the derivative of $M(t)$ with respect to $t$, one has

$$
\begin{aligned}
M^{\prime}(t) & =\int_{\Omega}|x|^{-s} u^{m} u_{t} \mathrm{~d} x \\
& =\int_{\Omega} u^{m+q} \mathrm{~d} x-\int_{\Omega}\left|\nabla u^{m}\right|^{p} \mathrm{~d} x \\
& =\frac{q-m(p-1)}{m+q} \int_{\Omega} u^{m+q} \mathrm{~d} x-p E(u) .
\end{aligned}
$$

If $q=m(p-1)$. From (8), one knows that $E(u)$ is nonincreasing with respect to $t$. Then, for any $t \geq 0$, one has

$$
M^{\prime}(t) \geq-p E\left(u_{0}\right)
$$

Integrating, one obtains

$$
M(t) \geq M(0)-p E\left(u_{0}\right) t, \quad \text { for } t \geq 0,
$$

which tells us that $M(t)>0$ since $M(0)>0$ and $E\left(u_{0}\right) \leq 0$; that is, the solution $u(x, t)$ of problem (1) does not possess extinction phenomenon.

If $q<m(p-1)$, with the help of Hölder's inequality, one gets

$$
\frac{q-m(p-1)}{m+q} \int_{\Omega} u^{m+q} \mathrm{~d} x \geq \frac{q-m(p-1)}{m+q} M^{(m+q) /(m+1)}(t)\left(\int_{\Omega}|x|^{s(m+q) /(1-q)} \mathrm{d} x\right)^{(1-q) /(m+1)}
$$


Exploiting (54) and (57), one can claim that

$$
M^{\prime}(t)-C_{4} M^{(m+q) /(m+1)}(t) \geq-p E\left(u_{0}\right),
$$

where

$$
\begin{aligned}
0>C_{4}= & \frac{q-m(p-1)}{m+q}\left(\int_{\Omega}|x|^{s(m+q) /(1-q)} \mathrm{d} x\right)^{(1-q) /(m+1)} \\
& >\frac{q-m(p-1)}{m+q} R^{s(m+q) /(m+1)}|\Omega|^{(1-q) /(m+1)}>-\infty .
\end{aligned}
$$

Since $M(0)>0$ and $E\left(u_{0}\right)<0$, then from (58) and Lemma 3, it follows that

$$
M(t) \geq \min \left\{M(0),\left(\frac{p E\left(u_{0}\right)}{C_{4}}\right)^{(m+1) /(m+q)}\right\}>0,
$$

which means that the solution $u(x, t)$ of problem (1) does not possess extinction phenomenon.

\section{Data Availability}

The data used to support the findings of this study are included within the article.

\section{Conflicts of Interest}

The authors declare that they have no conflicts of interest.

\section{Acknowledgments}

This paper was supported by the Natural Science Foundation of Hunan Province (Grant no. 2019JJ50160), Scientific Research Fund of Hunan Provincial Education Department (Grant no. 20A174), and Scientific Research Fund of Hunan University of Science and Technology (Grant no. KJ2123).

\section{References}

[1] D. M. Liu and C. L. Mu, "Cauchy problem for a doubly degenerate parabolic equation with inhomogeneous source and measure data," Differential and Intergral Equations, vol. 27, pp. 1001-1012, 2014.

[2] J. L. Vázquez, The Porous Medium Equation: Mathematical Theory, The Clarendon Press, Oxford University Press, Oxford, UK, 2007.

[3] Z. Q. Wu, J. N. Zhao, J. X. Yin, and H. L. Li, Nonlinear Diffusion Equations, World Scientific Publishing Co., Inc., River Edge, NJ, USA, 2001.

[4] D. Li, J. Liu, and L. Zheng, "A zero-dimensional valuation ring is 1-Gröbner," Journal of Algebra, vol. 484, pp. 334-343, 2017.

[5] Y. Liu and H. Su, "Some necessary and sufficient conditions for containment of second-order multi-agent systems with sampled position data," Neurocomputing, vol. 378, pp. 228237, 2020.

[6] C. J. Xu, H. C. Xu, H. S. Su, and C. Liu, "Disturbance-observer based consensus of linear multi-agent systems with exogenous disturbance under intermittent communication," Neurocomputing, vol. 404, pp. 26-33, 2020.

[7] Q. Xin, "Partial differential equation and its applications in image denoising and segmentation (in Chinese)," Ph.
D. thesis, Chongqing: College of Mathematics and Statistics, Chongqing University, Chongqing, China, 2011.

[8] C. J. Xu, B. F. Li, and L. Yang, "Semi-global containment of discrete-time high-order multi-agent systems with input saturation via intermittent control," IET Control Theory and Applications, vol. 14, pp. 2303-2309, 2020.

[9] Z. Chaouai and A. E. Hachimi, "Qualitative properties of weak solutions for $p$-Laplacian equations with nonlocal source and gradient absorption," Bulletin of the Korean Mathematical Society, vol. 57, pp. 1003-1031, 2020.

[10] Y. Chen, J. Wang, and H. Zhang, "Extinction for a couple of fast diffusion systems with nonlinear sources," Nonlinear Analysis: Real World Applications, vol. 14, no. 4, pp. 19311937, 2013.

[11] E. DiBenedetto, Degenerate Parabolic Equations, Springer, NewYork, NY, USA, 1993.

[12] Z. B. Fang and X. Xu, "Extinction behavior of solutions for the -Laplacian equations with nonlocal sources," Nonlinear Analysis: Real World Applications, vol. 13, no. 4, pp. 17801789, 2012.

[13] Y. Han and W. Gao, "Extinction for a fast diffusion equation with a nonlinear nonlocal source," Archiv der Mathematik, vol. 97, no. 4, pp. 353-363, 2011.

[14] Y. Han, H. Li, and W. Gao, "Extinction and non-extinction of solutions to a P-laplace equation with a nonlocal source and an absorption term," Mathematical Modelling and Analysis, vol. 19, no. 2, pp. 169-179, 2014.

[15] R. G. Iagar and P. Laurençot, "Positivity, decay, and extinction for a singular diffusion equation with gradient absorption," Journal of Functional Analysis, vol. 262, no. 7, pp. 3186-3239, 2012.

[16] M. Liao and W. Gao, "Blow-up phenomena for a nonlocal $p$ Laplace equation with Neumann boundary conditions," Archiv der Mathematik, vol. 108, no. 3, pp. 313-324, 2017.

[17] D. M. Liu and C. L. Mu, "Extinction for a quasilinear parabolic equation with a nonlinear gradient source," Taiwanese Journal of Mathematics, vol. 18, pp. 1329-1343, 2014.

[18] D. M. Liu and C. L. Mu, "Extinction for a quasilinear parabolic equation with a nonlinear gradient source and absorption," Journal of Applied Analysis and Computation, vol. 5, pp. 114-137, 2015.

[19] D. Liu and C. Mu, "Critical extinction exponent for a doubly degenerate non-divergent parabolic equation with a gradient source," Applicable Analysis, vol. 97, no. 12, pp. 2132-2141, 2018.

[20] D. M. Liu, C. L. Mu, and I. Ahmed, "Blow-up for a semilinear parabolic equation with nonlinear memory and nonlocal nonlinear boundary," Taiwanese Journal of Mathematics, vol. 17, pp. 1353-1370, 2013.

[21] D. M. Liu and L. Yang, "Extinction phenomenon and decay estimate for a quasilinear parabolic equation with a nonlinear source," Advances in Mathematical Physics, vol. 2021, Article ID 5569043, 7 pages, 2021.

[22] Y. Wang and J. Yin, "Critical extinction exponents for a polytropic filtration equation with absorption and source," Mathematical Methods in the Applied Sciences, vol. 36, no. 12, pp. 1591-1597, 2013.

[23] X. Xu and T. Cheng, "Extinction and decay estimates of solutions for a non-Newton polytropic filtration system," Bulletin of the Malaysian Mathematical Sciences Society, vol. 43, no. 3, pp. 2399-2415, 2020.

[24] J.-S. Guo, B. Hu, and B. Hu, "Blowup rate estimates for the heat equation with a nonlinear gradient source term," Discrete 
and Continuous Dynamical Systems - A, vol. 20, no. 4, pp. 927-937, 2008.

[25] P. Quittner and P. Souplet, Superlinear Parabolic Problems: Blow-Up, Global Existence and Steady States, Birkhäuser Verlag AG, Basel, Switzerland, 2007.

[26] J. L. Vázquez and P. Souplet, "Stabilization towards a singular steady state with gradient blow-up for a diffusion-convection problem," Discrete and Continuous Dynamical Systems, vol. 14, pp. 221-234, 2006.

[27] H. Yuan, X. Xu, W. Gao, S. Lian, and C. Cao, "Extinction and positivity for the evolution p-Laplacian equation with L1 initial value," Journal of Mathematical Analysis and Applications, vol. 310, no. 1, pp. 328-337, 2005.

[28] Y. G. Gu, "Necessary and sufficient conditions of extinction of solution on parabolic equations," Acta Math. Sinica, vol. 37, pp. 73-79, 1994, in Chinese.

[29] Y. Tian and C. Mu, "Extinction and non-extinction for a $p$ Laplacian equation with nonlinear source," Nonlinear Analysis: Theory, Methods \& Applications, vol. 69, no. 8, pp. 2422-2431, 2008.

[30] J. X. Yin and C. H. Jin, "Critical extinction and blow-up exponents for fast diffusive p-Laplacian with sources," Mathematical Methods in the Applied Sciences, vol. 31, pp. 1383-1386, 2007.

[31] C. Jin, J. Yin, and Y. Ke, "Critical extinction and blow-up exponents for fast diffusive polytropic filtration equation with sources," Proceedings of the Edinburgh Mathematical Society, vol. 52, no. 2, pp. 419-444, 2009.

[32] J. Zhou and C. Mu, "Critical blow-up and extinction exponents for non-Newton polytropic filtration equation with source," Bulletin of the Korean Mathematical Society, vol. 46, no. 6, pp. 1159-1173, 2009.

[33] Z. Tan, "Non-Newton filtration equation with special medium void," Acta Mathematica Scientia, vol. 24, no. 1, pp. 118-128, 2004.

[34] Y. Wang, "The existence of global solution and the blowup problem for some p-Laplace heat equations," Acta Mathematica Scientia, vol. 27, no. 2, pp. 274-282, 2007.

[35] J. Zhou, "A multi-dimension blow-up problem to a porous medium diffusion equation with special medium void," Applied Mathematics Letters, vol. 30, pp. 6-11, 2014.

[36] J. Zhou, "Global existence and blow-up of solutions for a nonNewton polytropic filtration system with special volumetric moisture content," Computers and Mathematics with Applications, vol. 71, no. 5, pp. 1163-1172, 2016.

[37] X. Deng and J. Zhou, "Global existence, extinction, and nonextinction of solutions to a fast diffusion p-laplace evolution equation with singular potential," Journal of Dynamical and Control Systems, vol. 26, no. 3, pp. 509-523, 2020.

[38] M. Badiale and G. Tarantello, "A sobolev-hardy inequality with applications to a nonlinear elliptic equation arising in astrophysics," Archive for Rational Mechanics and Analysis, vol. 163, no. 4, pp. 259-293, 2002.

[39] B. Guo and W. Gao, "Non-extinction of solutions to a fast diffusive $p$-Laplace equation with Neumann boundary conditions," Journal of Mathematical Analysis and Applications, vol. 422, no. 2, pp. 1527-1531, 2015.

[40] W. Liu and B. Wu, "A note on extinction for fast diffusivepLaplacian with sources," Mathematical Methods in the Applied Sciences, vol. 31, no. 12, pp. 1383-1386, 2008. 